\title{
Remote Monitoring Device Design of Water Quality Information for Rivers and Lakes Based on ARM Techniques
}

\author{
Jiping $\mathrm{Xu}$, Lingbin Wang, Jiabin $\mathrm{Yu}$, Xiaoyi Wang, and Junyang Yao
}

\begin{abstract}
In the context of rivers and lakes' environmental deterioration as well as the water bloom's explosion, an informative remote monitoring device of water quality is exploited for rivers and lakes. Based on the ARM Technique, serial communications technology, GPS global positioning technology, and GPRS/3G remote data communication technology are adopted to exploit a main controller of water quality monitoring which is made of input and output interfaces, analog parameter input interfaces, standard interface and Network Interface. The idea of modularization is used in constructing the software system combined with the YSI data acquisition system, security monitoring system, pumping water system, global positioning system (GPS). The practical running indicates that on the basis of collecting and transmitting the water quality information, the instrument can well meet the control requirements.
\end{abstract}

Index Terms-ARM Techniques, water quality monitor, GPRS/3G communication technology.

\section{INTRODUCTION}

With the intensification of global eutrophication, the phenomenon of blooms is becoming much more common. The outbreak of algal bloom is unexpected and once it occurs, the water body will be seriously stink, which will lead to a structural change and functional degradation to the ecological system that may bring greater losses or disasters to the human and the nature [1]. The existing methods of water quality monitoring system [2] can be divided into two categories: 1) Artificial operation is employed which consists of a large monitoring system whereas takes a long time for test process. 2) Online testing methods whose parameters of water quality testing are single that cannot transmit test data in real time [3]. Based on these two kinds of systems, the value received from is has a low representation which is unable to timely reflect the changes of the water pollution. Therefore, auto-water quality estimation and algae bloom forecasting and early-warning systems are equipped in the areas where the bloom is easy to break out. Besides, various water quality monitoring stations are installed in order to

Manuscript received April 13, 2013, revised July 12, 2013. This study is supported by the National Natural Science Foundation of China, Beijing Natural Science Emphases Foundation (51179002), Beijing New Star Project on Science and Technology (2010B007), Beijing municipal project of education commission (PXM2013 014213_00004). Those supports are gratefully acknowledged.

Jiping $\mathrm{Xu}$, Lingbin Wang, and Jiabin Yu are with Beijing Technology and Business University, College of Computer and Information Engineering, Beijing, China (e-mail: xujiping@139.com, wlb19890913@163.com, yujiabin_1984@163.com). obtain the information about the water quality by virtue of host system which can protect the environment to the maximum limit and do have a great significance to society and nature.

In recent years, ARM Techniques have been widely concerned possessing a very broad prospect. The device provided by this technology has the advantage of being cut easily, small in size, low cost and stable performance [4]. For this reason, applying this technique to the water quality monitoring, evaluation and the development of bloom forecast warning intelligent information system is also one of the key direction of future water environment research [5], [6].

A design method of remote monitoring instrument of water quality information based on ARM Techniques and GPRS/3G techniques [7] is presented in this paper. The whole framework of the monitor is introduced, the overall design scheme of instrument is presented, the hardware and software systems of monitor are designed and the rivers water quality information acquisition and transmission function is achieved, which could provide a basis for water environment protection, forecasting and warning. Then it will be illustrated amply in terms of the general design of instruments and main controller design (Including hardware design, software design, test analysis.).

\section{General Design of InStruments}

As is depicted in the Fig. 1, the framework of remote monitoring instrument of water quality information can be watched.

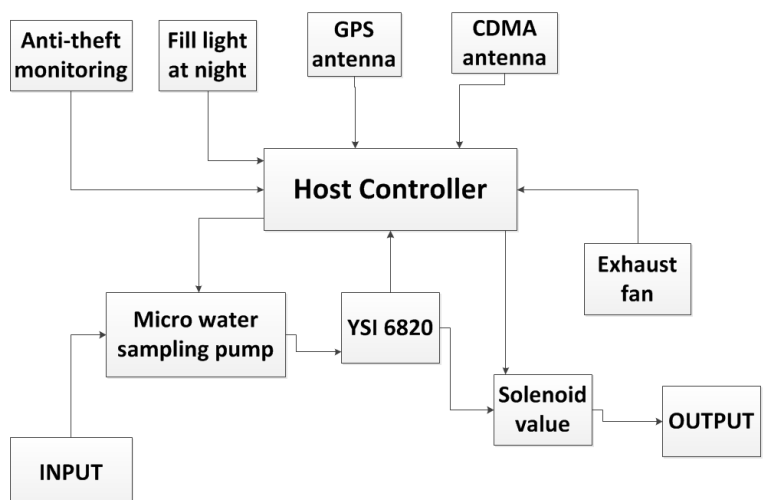

Fig. 1. Framework of remote monitoring instrument of water quality information

The monitoring is mainly composed of followed parts including YSI data acquisition system, remote monitoring system, pumping water system, global positioning system 
and ARM hardware control system. In this article, we will focus on Tangshan monitoring stations to specifically analyze the application of the system.

River Qinglong monitoring stations are equipped with YSI water quality sensors and online water quality monitor. Imported YSI 6820 multi-parameter water quality monitor is adopted for the water quality sensor. It is a multi-parameter instrument suitable for multipoint sampling measurement, long-term field monitoring and cross-section analysis, which can monitor as many as seventeen parameters at the same time. Users can set the time interval of data acquisition of the monitor whose stored readings can reach 150,000. The parameters collected from sensors, large amounts of data information received from GPS in real time have been achieved in each water quality monitoring station which could store data information within a certain time as well. Online water quality monitor which is packaged with information processing algorithm could analyze and process single point of monitoring data in advance as well as transmit the data to superior forecasting warning level periodically so as to realize the decentralized monitoring and centralized management function. At the same time, for monitoring objects with the characteristics of dry season, online water quality monitor can realize water quality monitoring and intelligent control with devices such as the seal water tank, micro pump and drainage devices solenoid drain valve. Aiming at the problems such as security, anti-theft, anti-destroyed, all devices are assembled on the high-performance monitoring steel cabinet and equipped with the security cameras, which can prevent from stealing by means of timely alarming and saving historical evidence.

Water areas monitored are extensive. The distribution of monitoring points is scattered. It is not suitable to monitor the water quality and make a warning by means of traditional wiring of monitoring which would invest a lot of cost on transmission. The problem of the remote transmission of information has been efficiently solved by the $3 \mathrm{G}$ remote transmission technology in this system. It is easy to transmit the huge amounts of data information collected by field monitoring layer to host server terminal and mobile client as well based on the technology mentioned above.

\section{PRIMARY CONTROLler Design}

In order to meet the requirements of River Qinglong lakes monitoring project, powerful ARM 9 series processor is chosen as the primary controller that has excellent properties such as low voltage low power consumption and high integration which can provide reliable and flexible solution for demanding and cost sensitive embedded application. Furthermore, the controller could reduce the chip area, complexity and power consumption which in turn boost up new products to market. This system with ARM9 processor can deal with a lot of water monitoring project data information more quickly, reduce intelligent operation time and improve the efficiency of the analysis and processing of the controller and the data transmission [8].

\section{A. Hardware Design}

The host controller of water quality information remote monitoring is composed primarily of input and output, analog input, RS232 interface, network interface, indicator light, 12 VDC output, 5 VDC input, 24 VDC input, the reset button, watchdog, extensible interfaces, GPRS/3G communication module and GPS receiving module. It will be viewed in the main function modules to introduce the hardware requirements and application in Tangshan project. As is shown in Fig. 2, the structure of hardware system can be watched.

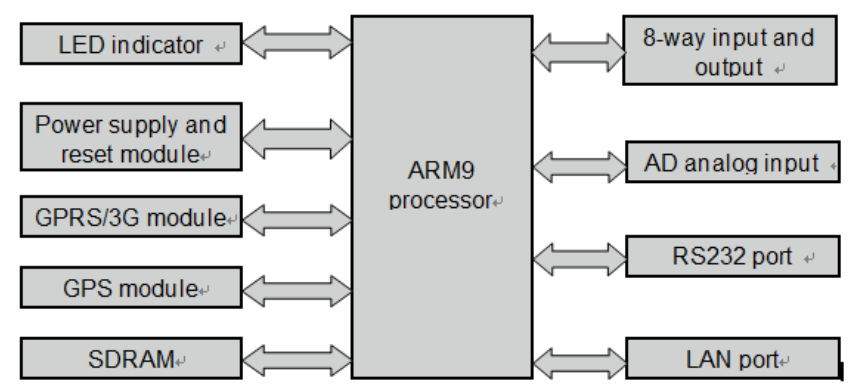

Fig. 2. Structure of hardware system

1) Input and output port is composed of the eight-way dry contact on-off input ( $5 \mathrm{~V}$ to $24 \mathrm{~V}$ in the way of direct-current) and four-way dry contact switch output $(5 \mathrm{~V}$ to $24 \mathrm{~V}$ in the way of direct-current and $5 \mathrm{~V}$ to $24 \mathrm{~V}$ in the way of alternative current) which are with certain pressure resistance. Considering ESD protection, terminal is used as the interface device in this system. Photoelectric detection switch Input 8 is the only one being used in Tangshan project. When there is an open or close action, switch input signal will be sent to the primary controller from Input 8 interface. When the signal is received by primary controller, the controller will open the light-compensating lamp and take pictures at once. The remaining seven switches serve as extensions to the input port while the four-way dry contact switches are relay, pump, fan and light-compensating lamp respectively.

2) The input range of two-way $\mathrm{AD}$ analog is $4 \mathrm{~mA}$ to 20 $\mathrm{mA}$ whose conversion precision is 8 bits, using connector as the interface device. Serving as the simulation input, hydraulic pressure sensor AD1 converts the pressure signal to current signal and realizes the detection of water tank liquid level while the temperature sensor AD2 converts temperature signal to current signal and realizes the control of fan's starting and stopping in order to ensure the appropriate temperature of the primary controller.

3) Two-way RS232 interface circuit is using a standard interface which needs only three interface lines when transmitting control signal, namely "sending data", "receiving data" and "signal ground". The nine core socket of DB-9 is adopted where the interface two is for receiving data, the interface three is for sending data and the interface five is for signal ground. Interference can be shielded effectively with twisted-pair cable as transmission line. One of the RS232 interface connects YSI 6820 water quality sensors to acquire various parameters, such as chlorophyll and dissolved oxygen in water while the other one connects the camera to collect image data.

4) The water quality information collected by YSI, the geographical coordinate's information collected by GPS and the image information collected by security surveillance camera will be sent to host system via $3 \mathrm{G}$ network. 


\section{B. Software Design}

The development of software design is based on hardware structure and application using the modular programming ideas to build the software system combined with YSI data acquisition system, security monitoring system, pumping water system and Global Positioning System (GPS) whose function is to be able to complete the required demand.

This system provides the function of the remote network access. Web Server can use it for remote access and view the parameters of the equipment status as well. Operators can configure parameters remotely including RS232 interface parameters, switch value corresponding relationship between input and output and analog quantity range parameter settings; the clock settings; the name of the device settings; configuration of the network parameters such as the native IP address, destination IP address, destination port number, subnet mask, gateway address and DNS and so on; the choice of network protocol; heartbeat interval settings to upload the heartbeat information regularly so as to manage the equipment.

First of all, we initialize the system and configure related parameters of GPRS text messaging module by sending the AT commands to the primary controller after power on. Secondly, we require network communication by means of the $3 \mathrm{G}$ module, match the IP address and port number of the remote monitoring center server and establish TCP network connection with the server to transmit and process data [9]. After completion of system initialization, the water pump and electromagnetic is controlled by the timer to pump and release water and then the YSI data can be adopted, processed and transmitted. At the same time, the state of opening the door or not can be detected via the system that three photos will be taken continuously, processed and transmitted when there is an opening move. As is depicted in Fig. 3, the flow chart of the software system can be watched.

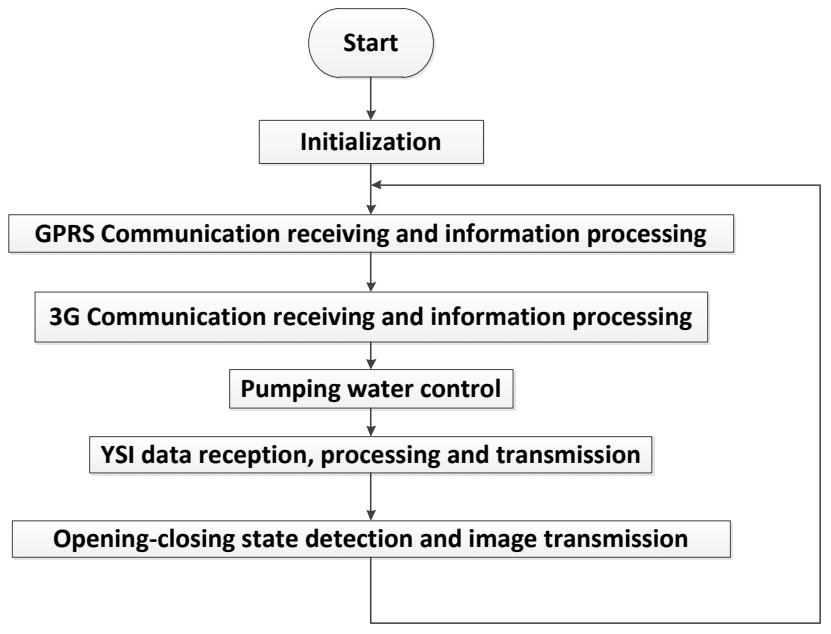

Fig. 3. Flow chart of software system

Among those functions, we will focus on pumping water control module to analyze logic and operation process of the project specifically in Tangshan. RTC timer in the program will minus one per second from one thousand and eight hundred second to zero second according to its own crystal frequency. When reducing to zero, namely every thirty minutes, the system starts pumping water. Firstly, electromagnetic valve will be opened pouring water for fifteen second until the original water in it is clean. Secondly, the pump will be opened to pump water for another fifteen second to flush water tank. Thirdly, electromagnetic valve will be closed to stop pouring water while only pumping at the same time to inject new water to the tank for fifteen second. Fourthly, the pump will be closed to stop pumping while the tank water is filled with the extend to full and YSI data can be collected. Whereby, the water tank is designed with the tapered shape with a port on the top side of the bearing. When the tank is filled with water while the pump is still open, the excrescent water will exclude from the drain outlet. As is shown in Fig. 4, the flow path can be watched.

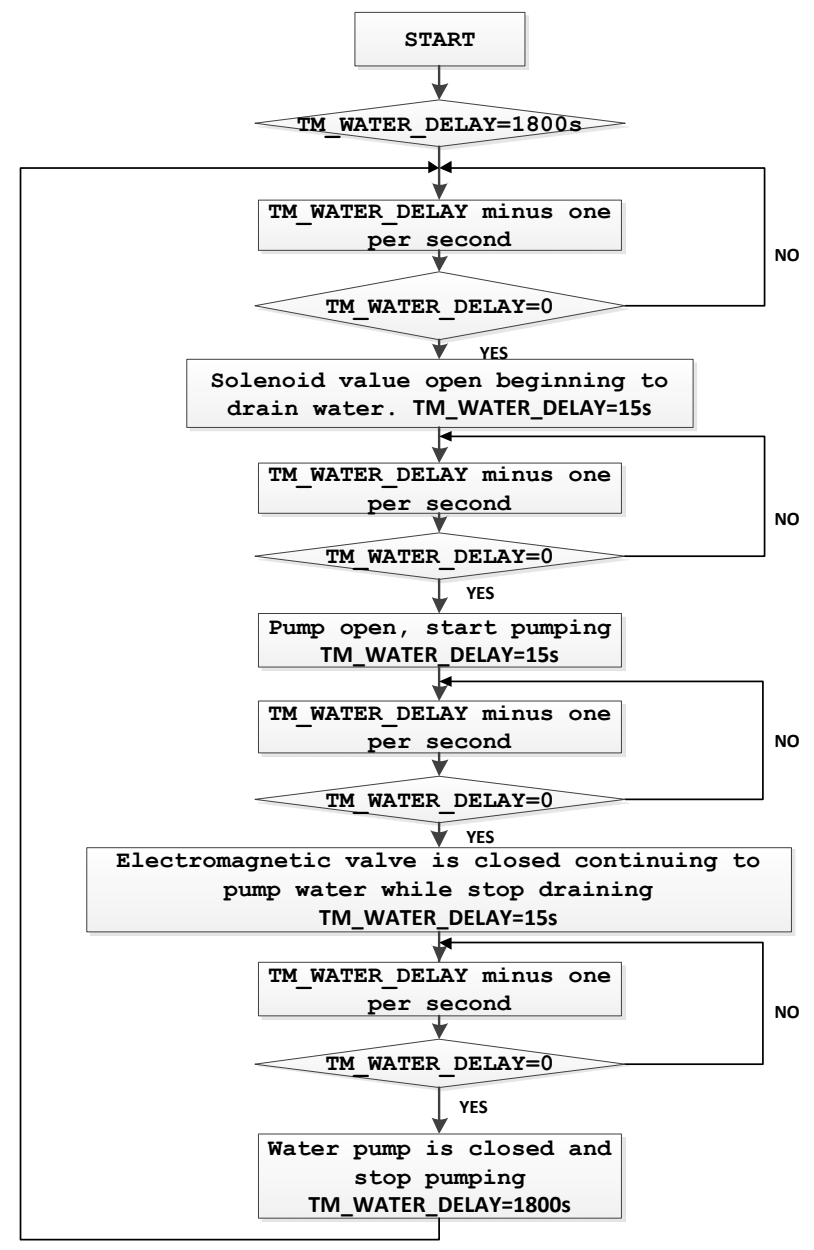

Fig. 4. Flow chart of pumping water control

\section{TEST ANALYSIS}

This system is tested under the laboratory condition where the YSI and image data are transmitted by means of the $3 \mathrm{G}$ network.

With serial debugging assistants, hardware's running status will be showed on the serial ports under this test which can provide reference basis and convenience for test personnel. When sending YSI data, the connection between $3 \mathrm{G}$ module and the host system is successful which will show "Net OK" indicating that system begins to send YSI data. After receiving the data successfully, the host system will return a piece of status information to the inferior when the thread will be closed meanwhile. But if there is no connection with host system for a long time, it will display "Time Out" and "Close Net" state .As is shown in Fig. 5, the serial port debug 
interface can be watched.

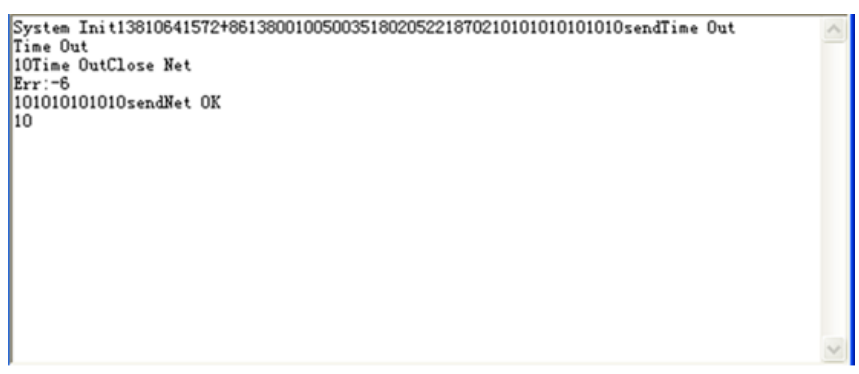

Fig. 5. Inferior serial debugging assistant

The upper forecasting warning system can get data transmitted from the inferior via the $3 \mathrm{G}$ network. What's more, it can make complex analysis and integrated intelligent management towards data in order to realize advanced features such as host controller algorithm parameter dynamic updates of remote monitoring, real-time data display, historical data query printing, eutrophication of water quality evaluation and long-term projections of water bloom, etc. In addition, GIS technology has been applied to the host system which can realize integrated water management of large area and water bloom dynamic color display. Furthermore, the host system configured with WEB information publish server can make the remote computers and mobile phones browse the site monitoring and analysis of information in real time.

The host and inferior test results show that YSI water quality information and image data transmission of the system is stable, which can better realize the collection processing and transmission function and improve the monitoring ability of lake water, effectively preventing the outbreak of water bloom.

\section{CONCLUSION}

Considering the problems that traditional river pollution monitoring means is much more complex, a kind of lake water information remote monitoring instrument based on embedded technology and GPRS / 3G technology is put forward. The structure design of inferior controller is reasonable which has realized the $3 \mathrm{G}$ transmission of the YSI data and images. This system can automatically access the GPRS / 3G network without network wiring with the advantages of wide coverage area and low cost that can monitor up to five measures of water quality according to different of pollution sources, which is convenient, flexible and with high precision. Installing the equipment on the bank can provide the basis for environmental protection management to work in full swing.

\section{REFERENCES}

[1] H. L. Liu, Control of Lake Eutrophication, Beijing, China environmental science press, pp. 2-3, 2011.

[2] W. Y. Tong, C. M. Liu, and G. C. Zhao, "The water quality parameters monitoring system of data remote traffic based on GPRS," Automation and Instrument, pp. 52-55, July 2010.

[3] C. Liuong, "Design of automated monitoring system of water quality PH based on GPRS," M.S. thesis, Jilin University, China, 2007.

[4] S. Nan, The principle and development technology application of embedded system; Beijing: Higher Education Press, 2008.

[5] J. Zai, Z. W. Liu, X. Y. Wang, and J. P. Xu, "The River Water Environmental Monitoring and Water Bloom Prediction System Based on Wireless Communication Technology," in System Simulation
Technology and application of Academic Conf., Jilin, 2010, pp. 504-507.

[6] X. Y. Wang, J. Liu, Z. W. Liu, and J. P. Xu, "A Synthesis Evaluation Method on Water Eutrophication Based on Fuzzy Optimzation-MAMD," in Proc.Twenty-ninth Chinese Control Conf., Beijing, 2010, pp. 1303-1306.

[7] J. Zhang and C. Gai, "The Application of GPRS Radio Communication Technology in the Online Envirorment Monitoring," Modern Agricultural Science and Technology, pp. 260-261, May 2009.

[8] X. Y. Fan, M. X. Zhang, and D. L. Wang, "Introduction of the Embedded System," Techniques of Automation and Applications, February 2008.

[9] L. K. Zhang, The application of Design and development technology based on Visual Basic 6.0. Beijing: Posts \& Telecom press, 2004, pp. 402-416.

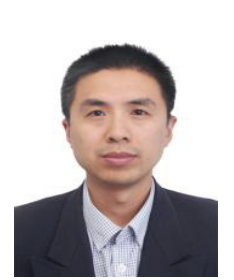

Xu Jiping was born in Hunan Province of China in April, 1979. A doctor degree in Engineering from Beijing Institute of Technology of China is received in July, 2008, majoring in systems engineering. The current major field is the key technology of IOT system about the remote monitoring of water environment etc.

He has been engaged in the job in the field of education, scientific research in Beijing Technology and Business University from July, 2008. The present title is Associate Professor. These years about forty academic paper have been published including. What he is interested in right now is the research of water environmental monitoring in lake and online cloud services of experts.

Dr.Jiping is the director of the Beijing institute of automation.

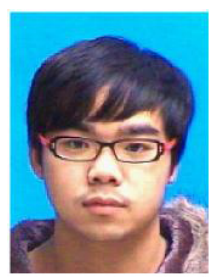

Wang Lingbin was born in Zhejiang Province of China in September 13th, 1989. The bachelor is received from Beijing Technology and Business University of China in 2012, majoring in automation. Master's degree is to be granted in 2015. The current major field is detection technology and automatic equipment.

$\mathrm{He}$ is a postgraduate right now who is interested in the research about water monitoring.

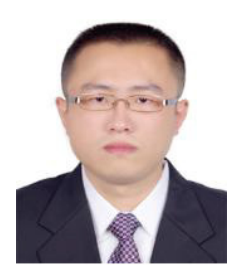

Yu Jiabin was born in Shandong Province of China in March, 1984. A doctor degree in Engineering from Chinese Academy of Sciences Institute of Automation is received in July, 2012, majoring in control theory and control engineering. The current major field is the key technology of high power electric spindle motor etc.

He has been engaged in the job in the field of education and scientific research in Beijing Technology and Business University from July, 2012.The present title is lecturer. What he is interested in right now is the research of water environmental monitoring in lake and data fusion.

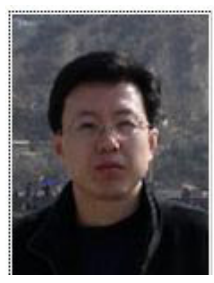

Wang Xiaoyi was born in Shanxi Province of China in June, 1975. A doctor degree in Engineering from Beijing Institute of Technology of China is received in July, 2006, majoring in control theory and control engineering. The current major field is the modeling of complicated water environment and optimal control of wastewater treatment etc.

$\mathrm{He}$ has been engaged in the job in the field of education, scientific research and management in Beijing Technology and Business University from July, 2006.The present title is Associate Professor. These years about eighty academic paper have been published including "the research based on the fuzzy Petri net optimal modeling of formation mechanism of algae blooms" published in Acta Electonica Sinica and "the cyanobacteria formation mechanism of water in cities" published in CIESC Journal etc. What he is interested in right now is the research of water environmental monitoring in lake, forecast of algal bloom and decisions of emergency management.

Dr. Xiaoyi is the committee member of the 8 th Beijing municipal association for science and technology as well as the director of the Beijing institute of automation. 